\title{
Non-singular black holes interiors need physics beyond the standard model
}

\author{
Ram Brustein ${ }^{(1,2)}$, A.J.M. Medved ${ }^{(3,4)}$
}

(1) Department of Physics, Ben-Gurion University, Beer-Sheva 84105, Israel

(2) Theoretical Physics Department, CERN, 1211 Geneva 23, Switzerland

(3) Department of Physics \& Electronics, Rhodes University, Grahamstown 6140, South Africa

(4) National Institute for Theoretical Physics (NITheP), Western Cape 7602, South Africa

ramyb@bgu.ac.il, j.medved@ru.ac.za

\begin{abstract}
The composition as well as the very existence of the interior of a Schwarzschild black hole (BH) remains at the forefront of interesting, open problems in fundamental physics. To address this issue, we turn to Hawking's "principle of ignorance", which says that, for an observer with limited information about a physical system, all descriptions that are consistent with known physics are equally valid. We compare three different observers who view the $\mathrm{BH}$ from the outside and agree on the external Schwarzschild geometry. First, the modernist, who accepts the classical BH as the final state of gravitational collapse, the singularity theorems that underlie this premise and the central singularity that the theorems predict. The modernist is willing to describe matter in terms of quantum fields in curved space but insists on (semi)classical gravity. Second is the skeptic, who wishes to evade any singular behavior by finding a loophole to the singularity theorems within the realm of classical general relativity (GR). The third is a postmodernist who similarly wants to circumvent the singularity theorems but is willing to invoke exotic quantum physics in the gravitational and/or matter sector to do so. The postmodern view suggests that the uncertainty principle can stabilize a classically singular $\mathrm{BH}$ in a similar manner to the stabilization of the classically unstable hydrogen atom: Strong quantum effects in the matter and gravitational sectors resolve the would-be singularity over horizon-sized length scales. The postmodern picture then requires a significant departure from (semi)classical gravity, as well as some exotic matter beyond the standard model of particle physics (SM). We find that only the postmodern framework is consistent with what is known so far about $\mathrm{BH}$ physics and conclude that a valid description of the $\mathrm{BH}$ interior needs matter beyond the SM and gravitational physics beyond (semi)classical GR.
\end{abstract}




\section{Introduction}

What is the final state of matter that collapses under its own gravity? The answer has long been debated, as far back as the early days of Newtonian gravity. When general relativity (GR) came along, the debate raged on, but now in terms of the framework of the new theory. Finkelstein eventually realized that the Schwarzschild solution was describing an event horizon [1] and was later revealed to be truly singular. Many attempted to prove otherwise and show that a singularity cannot be part of the correct description of the final state of collapsing matter. A period of intense debate then ensued between two opposing camps: Those unwilling to accept the demise of physics by its own hands, such as Wheeler [2], versus the likes of Buchdahl [3], Chandrasekhar [4, 5] and Bondi [6], who applied the equations of GR to show that "normal" matter cannot be stable when confined to some minimal radius. In parallel, more formal efforts at proving the inevitability of singularities were pursued by Raychaudhuri [7], Komar [8] and others. The debate ended (or so it seemed) when Penrose and Hawking proved their singularity theorems [9, 10]. Soon thereafter, the term "black hole" was invented and some semiclassical aspects were added by Bekenstein [11] and Hawking [12, 13]; thus establishing the paradigm of black hole (BH) thermodynamics and marking the beginning of the modern era of $\mathrm{BH}$ physics.

From the modern perspective, an observer should see nothing unusual as she falls through a $\mathrm{BH}$ horizon; after all, this is a region of weak gravity for a $\mathrm{BH}$ that is sufficiently massive. 11 This seemingly uncontroversial opinion is dependent on at least two implicit assumptions; namely that our normal understanding of spacetime geometry persists into the $\mathrm{BH}$ interior and there is a separation of scales between

\footnotetext{
${ }^{1} \mathrm{~A}$ large Schwarzschild BH in a four-dimensional, asymptotically flat spacetime is assumed throughout.
} 
the central singularity and the horizon at the gravitational radius. Although both seem reasonable enough contentions, there are no grounds for insisting that they must be true. The BH interior is by definition causally separated from the rest of the Universe, and there are reasons to suspect that the modern perspective is amiss, as it invokes a central singularity, as well as a reversal of time and space.

If there is no clear-cut picture of the interior, then what is one to do? Hawking implicitly answered this question long ago with a brief discussion on what he called the "principle of ignorance" [13]. To paraphrase, an observer who is lacking information about a physical situation is free to adopt any explanation that is consistent with the laws of physics and experimental data, as all such descriptions are equally likely. With the adoption of this principle, the portrait of a $\mathrm{BH}$ in $\mathrm{GR}$ is as valid as one's tolerance of singular regions of spacetime. And, as discussed below, a regularized singularity, the frequently prescribed remedy, is just as flawed as a mathematical infinity.

The task of constructing a viable model for the interior becomes all the more challenging when quantum effects are incorporated; in particular, the quantum process of $\mathrm{BH}$ evaporation [12. It has long been understood that an evaporating $\mathrm{BH}$ - for which the final outcome appears to be a maximally mixed state of thermal radiation - is inconsistent with the quantum principle of unitary evolution [13]. There was a time when the preferred way out of this dilemma relied upon a generalized notion of Bohr-like complementarity [14, 15, 16], which claimed that causally separated observers need not agree on physical events as long as they can never compare their observations. And so, on this basis, the same information can simultaneously be both inside and outside of a BH simply because no single observer could verify this duplicity. (If she could, this would contradict a quantum theorem against cloning.) Our own current view (see below) is that the interior observer gets to have no say 
and that this is how observer complementarity can be set aside.

Nevertheless, it has since been made clear that, given a unitary evaporation process, a single external observer can still see a violation of yet another cherished principle of quantum mechanics: the monogamy of entanglement or, as it is more formally known, the strong subadditivity of the von Neumann entropy. This conflict has been brought to light in [17, 18, 19, 20, 21] and, most famously, by the authors of [22] (AMPS). They "doubled down" on the GR model by moving the $\mathrm{BH}$ singularity all the way out to where the horizon should be, thus providing the BH with a metaphorical "firewall". To be fair, AMPS were not as much advocating for the existence of a firewall as they were illustrating the fallacy of using observer complementarity (at least in this context).

The advent of the firewall argument marked the beginning of the postmodern era of BH physics. The essence of the firewall argument is that it is not reasonable to simply ignore the interior and the ensuing challenge for the postmodern era is, succinctly, the singularity.

There has since been a long line of attempts at circumventing the AMPS solution while, at the same time, having one's unitary evolution and strong subadditivity too. (See [23] for an already exhausting list of what is but a small fraction of the ensuing papers.) More often than not, the singularity is treated as a triviality under the premise (sometimes implicit) that quantum gravity or string theory should somehow regularize any infinities and thus save the day. An additional implicit assumption is that the regularized singularity will not affect the structure of space time on horizonsized scales. The problem with this mindset is that a regularized singularity is no more or less than a BH remnant, as both imply an extremely large amount of stored information is a small region of space. And, although arguments for remnants still turn up from time to time, it is widely accepted that their putative existence opens 
up the Pandora's box of a destabilized vacuum [24] from which one should promptly move on. For a recent review on remnants, see [25].

A notable exception is Mathur's string-theory-inspired fuzzball model [26] (also, [27]), which considers a quantum superposition of singularity-free and horizon-free microstates. The total wavefunction is then supposed to mimic the properties of a semiclassical BH. Another exception is 't Hooft's BH without an interior [28, 29], which is based on the idea that high-energy incoming particles can be traded for lowenergy outgoing particles (these being quantum clones of one another), meaning that the interior need never be probed. Yet another exception is the graviton-condensate model of Dvali and Gomez [30], who invoke a highly-occupied state of gravitons as the state of the $\mathrm{BH}$ to obtain a regular interior with only an approximate horizon.

Meanwhile, another issue with BH evaporation has recently emerged; what Mathur has called the BH "causality paradox" 31. The challenge here is to explain how information can escape out of a $\mathrm{BH}$ given that the future light cone of any interior particle is completely contained within the horizon. This presents an additional hoop to jump through for those with a vested interest.

From our point of view, the singularity/remnant problem, the tension between strong subadditivity and unitarity, and the apparent conflict with causality represent different sides of the same three-sided coin. As long as there is a singularity, then information loss is inevitable because absorption by the singularity is effectively the same as passing through to another universe as in Wheeler's bag-of-gold spacetime 32. Conversely, any model that is based upon the rules of quantum theory and devoid of singularities must, by construction, respect unitarity, strong subadditivity and causality, as well as any other fundamental tenet of quantum theory. If gravitational collapse can somehow be avoided, then the rest will follow.

Our proposed resolution is that the uncertainty principle can stabilize a classically 
singular BH in essentially the same way that it can do so for a classically unstable atom. For an early discussion of this idea see Sect. 1.3 of [33]. We expect strong quantum effects from the matter and gravitational sectors to "smear" the would-be singularity over horizon-sized length scales. The self-consistency of this picture of the interior requires a significant departure from (semi)classical gravity, as well as some exotic matter which is outside the realm of the standard model (SM). The resulting picture is that of a "quantum star" that looks from the outside just like a BH.

But let us circle back to the beginning of the discussion and recall that one story is as good as another as long as it consistent with what is already known about physics. In light of this, we would like to understand the interior from the perspective of an external observer. The distinction between interior and exterior observers is more than semantics because different perspectives could have provided, in principle, complementary descriptions (in the quantum sense) of the same physical system. For instance, if Alice probes a system with operators that are non-commuting with respect to Bob's, this ubiquitous pair of observers will necessarily have conflicting descriptions. The essential difference between BHs and most other systems is that, in the $\mathrm{BH}$ case, the perspective of an external observer is the only one that really counts. This is, in our opinion, the essence of why $\mathrm{BH}$ complementarity as it is usually interpreted is problematic; the interior observer gets to have no say in the matter.

With all of this in mind, our objective is to describe the $\mathrm{BH}$ interior from the perspective of three different outside observers, each with their own distinct narrative. These will be (1) the modernist who adheres to the modern view of a $\mathrm{BH}$, that of GR including BH thermodynamics, and is willing to accept a (regularized) singularity as part of her worldview and therefore abandon unitarity, (2) the skeptic who allows exotic matter if its inclusion evades gravitational collapse, views BH thermodynamics 
and unitarity as desirable although non-essential but otherwise abides by the rules of classical GR and (3) the postmodernist who abhors a singular gravitational collapse, insists on unitarity and will call upon exotic physics in both the matter and gravitational sector as needed to complete a paradox-free picture.

We discuss each of these in turn and then provide an overview at the end.

\section{The modern perspective}

Here, we will describe the viewpoint of an external observer who insists on the BHs of GR as a starting point, while incorporating the commonly accepted ideas from the realm of semiclassical physics. This observer assumes gravitationally collapsing matter in the usual sense, except that the endpoint is some sort of regularized singularity or remnant. The firewall model would also fall under this category, as it at least starts out with the traditional (semiclassical) picture, and nothing unusual has to happen until the midpoint of $\mathrm{BH}$ evaporation as measured in units of diminishing entropy [22] (what is known as the Page time [34]).

As this picture of a $\mathrm{BH}$ is (almost) singular by design, the observer must be assuming some notion of a remnant is consistent with known physics. This observer must also accept the separation of scales between the singularity (or some regularized version of it) and the horizon, so that the former scale has no bearing on the latter. Although such a stance is highly questionable at best, we will assume for the sake of discussion that these are acceptable conditions and proceed to consider other aspects of the modernist's description. A Penrose diagram depicting the geometry (excluding evaporation) from the modern perspective is depicted in Fig. 1.

In view of the causality paradox, the observer must be of the impression that neither matter nor information could escape from the $\mathrm{BH}$ interior. If the observer 
still insists on BH radiation, as well she should, the obvious explanation is an external pair-production process just as Hawking described it in [13]. For any given pair, the positive-energy partner transitions into an emitted Hawking particle whereas the negative-energy partner passes through the horizon and consequently lowers the mass of the BH. There is, however, a wrench in the pair-producing gears: One must inevitably choose between either entangled pairs at the horizon but with an accompanying loss of information or, else, the purification of the radiation but at the prohibitive cost of a firewall, which is tantamount to moving the singularity all the way to the horizon. This choice suggests that the separation of BH scales may not be such an valid idea after all.

One way out of this conundrum is if the negative partner's "half" of the entanglement could be teleported or swapped to the external radiation; a protocol that was recently outlined in [35]. The good news is that, just like any other teleportation event, there can be no violation of causality. The not-so-good news is that, in this case, the swap is to take place just when the negative partner is to be annihilated, which would be much more tenable if it did not involve the BH singularity. And even though there has been a proposal to this effect - namely, the final-state solution [36] - it has been revealed to be a non-unitary process unless there is a disturbing amount of fine-tuning [37].

Another way out would be via a traversable wormhole in the case of a two-sided $\mathrm{BH}$ or, more generally and by the same logic, the $\mathrm{ER}=\mathrm{EPR}$ proposal [38]. In the latter scenario, the interior of the $\mathrm{BH}$ is connected to the external radiation via of an Einstein-Rosen bridge with multiple exits; any of which could be far away from any of the others in terms of real space. This bridge maintains the near-horizon entanglement as long as necessary while providing a conduit for the information to eventually flow out. It is, in fact, not totally clear whether or not the $\mathrm{ER}=\mathrm{EPR}$ 
picture falls more in line with postmodernist reasoning because, when the length of the ER bridge reaches the order of the horizon radius, the separation-of-scales assumption is no longer applicable (as would also be true for any postmodern model). On the other hand, the original proponents of $E R=E P R$ suggest that it provides an alternative interpretation of the seminal firewall model (see Sections 4.3 and 6 in [38]), and so we also include it here.

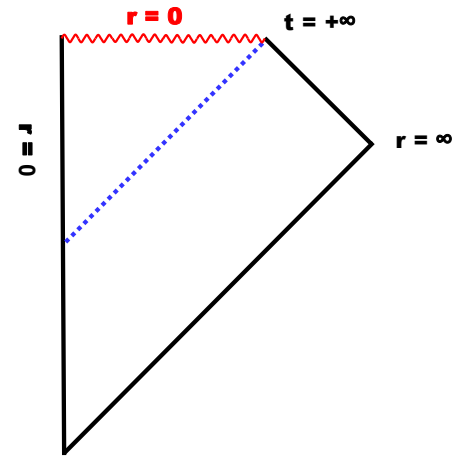

Figure 1: Penrose diagram of the modernist view of the $\mathrm{BH}$, excluding evaporation.

In any event, insofar as the Hawking particles originate strictly on the outside of the horizon, the associated entropy would have to be attributed to the thermal atmosphere of the BH. As shown by 't Hooft [39], this entropy would indeed agree with the $\mathrm{BH}$ area law [11, 12]. Nevertheless, the actual BH entropy would have to be located in the singularity, as this is where all the collapsed matter ends up. And so the modernist's story takes us right back to the remnant idea and its associated failings. In short, one must be willing to suspend disbelief to conclude that this 
problematic description of the interior is consistent with the known laws of physics.

\section{The skeptic's perspective}

As already stated, this would be the viewpoint of an external observer who proposes to avoid collapse by incorporating exotic matter but, at the same time, wants to stay within the realm of classical GR. The exotic matter need not be an actual form of matter; for instance, it could be some classical terms in the stress tensor that are meant to mimic corrections from quantum gravity. Regardless, because of the adherence to GR, any such observer will have to confront the Buchdahl bound [3].

Buchdahl considered the stability (or lack thereof) of a spherically symmetric matter distribution. Implementing only causality and a handful of common-sense assumptions about the energy density $\rho$ and pressure $p$, he was able to establish that stability required a minimal radial size of 9/8 Schwarzschild radii. Even though our focus will be on Buchdahl's calculation, similar reasoning led to the same general conclusions in some contemporary articles and the same basic principles are the essence of the famous singularity theorems (see the Introduction).

What was for a long time overlooked, although noticed and then summarily dismissed by Bondi [6], is that a maximally negative radial component of pressure, $p_{r}=-\rho$, is sufficient to bypass the Buchdahl bound, provided that the matter remains sufficiently dense all the way up to the Schwarzschild radius (this caveat will be clarified below). But it was eventually realized that negative pressure is indeed a

key ingredient for evading the Buchdahl bound (as well as the singularity theorems) and has since been incorporated into many attempts at modeling the interior of a regularized BH. Generically, such models are based on the idea that the deviations from GR only occur at scales where quantum-gravitational corrections become im- 
portant. These models describe objects for which the deviations are limited to some scale $R_{Q G} \ll R_{S}$, where $R_{S}$ is the object's Schwarzschild radius.

A prototype model of this kind is that of Hayward [40]. The geometry in this model is defined via the line element

$$
d s^{2}=-f(r) d t^{2}+\frac{1}{f(r)} d r^{2}+r^{2} d \Omega_{2}^{2},
$$

for which

$$
f(r)=1-\frac{2 m r^{2}}{r^{3}+2 m L^{2}},
$$

and the corresponding Penrose diagram is shown in Fig. 2. The length scales $m$ and $L$ determine the location of a pair of a horizons; an outer one at $r \sim 2 m$ and an inner horizon at $r \sim L$ (the latter also plays the role of $R_{Q G}$ ). The Hayward model features a negative radial pressure $p_{r}=-\rho$ and a transverse pressure $p_{\perp}$ that is negative near the center but positive for $r>L$. The energy density and pressure are both parametrically small outside of the inner horizon.

The Frolov-Markov-Mukhanov (FMM) model employs the same basic idea as Hayward's, but with the inner horizon replaced by a shell which connects a truncated Schwarzschild interior to an entire de Sitter universe in a smooth way [41]. The Penrose diagram corresponding to this geometry is illustrated in Fig. 3. Other models of this ilk - exploiting the idea of negative pressure for the purpose of regularizing the interior - are the gravastar [42], the black star [43], a back-reaction-corrected geometry [44] and our own model, the collapsed polymer BH [45], as well as [46, 47, 48]. See [49, 50, 51] for extensive reviews of models of regular BHs. Apparently, the earliest example of a non-singular BH can be attributed to Bardeen [52, who rather based his model on a core of charged matter. A recent version of Bardeen's model can be found in [53], which has the same causal structure as that of the Hayward model. 


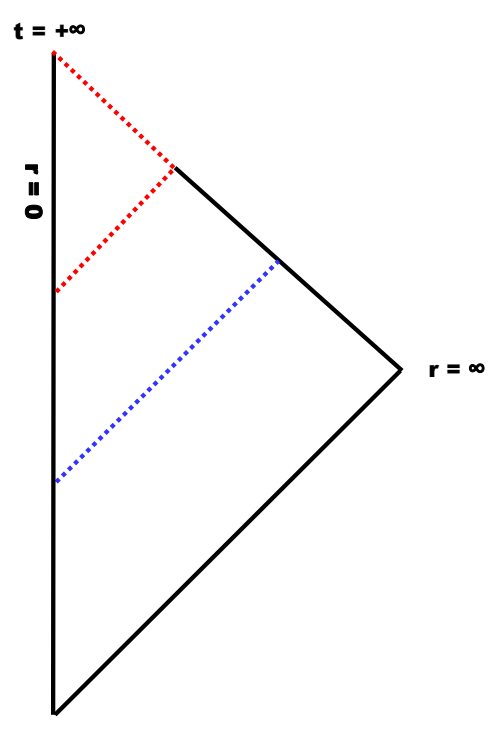

Figure 2: Penrose diagram of the Hayward model of a regular $\mathrm{BH}$, representing a skeptic's perspective.

But there is a major issue with most of these regularized $\mathrm{BH}$ models that comes to the fore when the evaporation process is taken into account; the earliest examples of which can be found in [54, 40]. As explained in [55, 56], it is a generic feature of regular $\mathrm{BH}$ solutions with $R_{Q G} \ll R_{S}$ that the energy of their emitted particles can sum up to an amount which is much larger than the object's original mass. Of course, if one is willing to ignore quantum mechanics completely, the problem goes away. We do, however, view this as an indication that such models are fundamentally inconsistent.

What is then needed is an object whose interior metric never approaches the Schwarzschild solution until it is parametrically close to the horizon; that is, $R_{Q G} \sim$ $R_{S}$. From this perspective, our own polymer model and the gravastar stand out because, in these two cases, the deviations persist up to a radius that is parametrically 


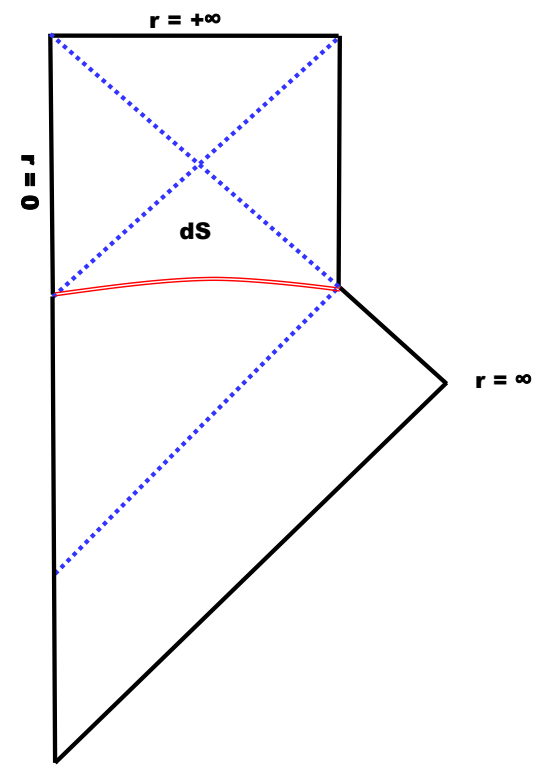

Figure 3: Penrose diagram of the FMM model of a regular BH, representing another perspective of a skeptic.

close to the outer surface [42, 45]. We will then turn our attention to this pair of models, beginning with the gravastar.

For Mazur and Mottola's gravastar model [42], the interior has a constant energy density and a constant, isotropic pressure [57]. This solution describes an ultracompact object with what is essentially a de Sitter interior along with an outer shell of matter. The latter is necessary to ensure that the de Sitter interior can be matched smoothly to the exterior Schwarzschild solution. The interior geometry of this model is described by the line element

$$
d s_{i n t}^{2}=-\frac{1}{4}\left(1-\frac{r^{2}}{R_{S}^{2}}\right) d t^{2}+\frac{1}{1-\frac{r^{2}}{R_{S}^{2}}} d r^{2}+r^{2} d \Omega_{2}^{2}
$$

and the Penrose diagram for the gravastar is presented in Fig. 4 .

Our own proposal — the collapsed-polymer model [58] - similarly has a maxi- 


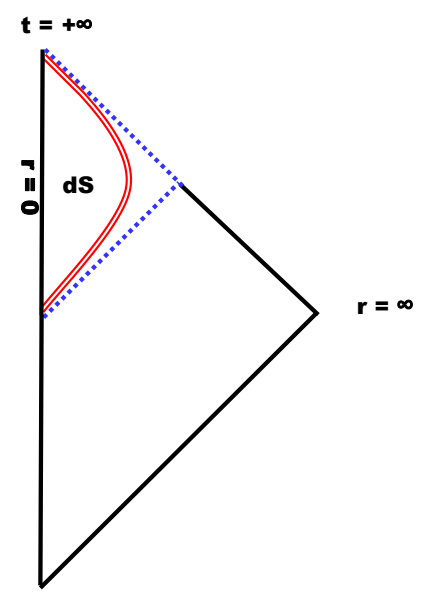

Figure 4: Penrose diagram of the gravastar model of a regular $\mathrm{BH}$, representing yet another skeptic perspective.

mally negative pressure, but only from the perspective of a skeptic. This subtle point regarding observer dependence was touched upon in [45] and will be elaborated on later when we discuss the same model from a postmodern perspective. Meanwhile, as far as the skeptic is concerned,

$$
p_{r}=-\rho=-\frac{1}{8 \pi G r^{2}}
$$

along with a vanishing transverse pressure [45]. 2 A model that features $p_{r}=-\rho \propto$ $-\frac{1}{r^{2}}$ was discussed in another context [59, 60] in which the negative pressure was sourced by a spherically symmetric "hedgehog" configuration of cosmic strings.

The interior line element of the skeptic's version of the polymer geometry is given

\footnotetext{
${ }^{2}$ In spite of appearances, this is a non-singular matter distribution as the physically relevant quantity, $d r 4 \pi r^{2} \rho$, is finite throughout.
} 
by

$$
d s^{2}=-f(r) d t^{2}+\frac{1}{f(r)} d r^{2}+r^{2} d \Omega_{2}^{2}
$$

such that $f(r)=0$. The metric then adopts the peculiar form of $g_{t t}=g^{r r}=0$ throughout the interior; implying that the whole region is null. Every spherical surface of constant radius is itself just like a BH horizon! The Penrose diagram corresponding to this model is depicted in Fig. 5.

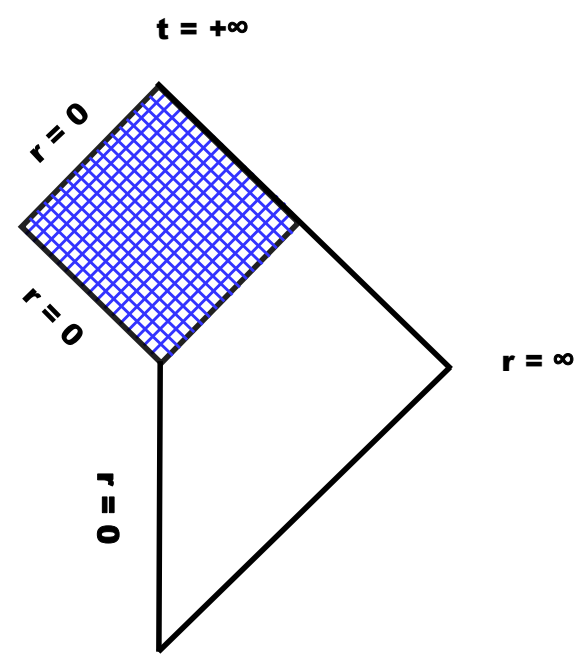

Figure 5: Penrose diagram of the polymer model of a regular BH from a skeptic's perspective. It should be viewed, as explained in [61], as part of an extended diagram. The whole interior is null, each sphere is like a BH horizon. See Fig. 6 for the postmodern perspective of the same model.

A remarkable aspect of the skeptic's polymer model is its complete stability 
against quantum perturbations [45]; meaning that there is no opportunity for the spontaneous emission of Hawking radiation. This is consistent with having zero entropy, as a classical solution should. This is fine with the skeptic since his preference would be to rely on classical gravitational physics as much as possible, even if certain terms in the Lagrangian are meant to imitate the effects of quantum gravity.

Nevertheless, an external perturbation that violates the null energy condition (as is perfectly reasonable in a quantum theory ${ }^{3}$ will produce radiation, albeit in the form of gravitational waves, and also some modified Hawking radiation [62, 63]. And, because of this violation, faster-than-light travel can be anticipated, as discussed recently in [64]. The causality paradox is then evaded in this case in a way that does not contradict the SM or quantum physics. In fact, as explained in the next section, the external perturbation does not need to violate any energy condition at all for the purposes of bypassing the causality bound. Nevertheless, according to a skeptic, an external source is indeed necessary for the polymer model because of the otherwise stable interior.

The way to understand the previous claim of faster-than-light travel is to notice that light-like travel of incoming/outgoing perturbations can be expected in a medium for which $p_{r}^{2}=\rho^{2}$ holds identically because then the radial speed $v_{r}$ is unity, $\frac{v_{r}^{2}}{c^{2}}=\left|\frac{d p_{r}}{d \rho}\right|=1$, and that the null version of the Raychaudhuri equation (along with Einstein's equations) tells us that light rays will defocus only if the null energy condition is violated. This becomes an "if and only if" statement provided that the associated shear, torsion and expansion $\Theta$ are all small enough to neglect because, in this case,

$$
\frac{d \Theta}{d \lambda}=-\left(\rho+p_{r}\right)>0,
$$

\footnotetext{
${ }^{3} \mathrm{~A}$ quantum description of an exterior matter system is acceptable to the skeptic.
} 
where $\lambda$ is the relevant affine parameter.

Irrespective of the causality paradox, this perspective of the interior still presents a significant problem: the conspicuous absence of an entropy, at least one that is large enough to account for the $\mathrm{BH}$ area law. This state of affairs applies just as well to the gravastar as it does to the skeptic's version of the polymer $\mathrm{BH}$, and quite possibly to any other self-consistent model with a classical and regularized interior. To resolve this issue, one could resort to treating the entropy as a mechanical quantity, in much the same way that the Wald entropy is introduced [65]. This is, in fact, the approach that was adopted for the gravastar model in [57]. Meanwhile, the vanishing of the thermodynamic entropy is necessarily true given that $T s=\rho+p=0$ as it is for the gravastar or, in the case of the skeptic's polymer model, $T s=\rho+p_{r}=0$, 4 where $s$ is the entropy density and $T$ is the temperature. The alternative of a vanishing temperature would be equally unwelcome. And so, even though there may be an emission of radiation, its temperature would not have to agree with the standard paradigm of $\mathrm{BH}$ thermodynamics.

In summary, the skeptic's story is fine at the level of classical gravitational physics but is ill suited for the incorporation of quantum effects. Indeed, the various models of regularized $\mathrm{BH}$ solutions are fine as long as evaporation and other related quantum aspects are not considered, as previously discussed. However, when one attempts to include quantum effects, inconsistencies abound.

\footnotetext{
${ }^{4}$ The reason that $p_{r}$ is used here is that $\mathrm{BH}$ horizons effectively act like $(1+1)$-dimensional surfaces and every spherical surface in this interior acts like a horizon. Alternatively, the postmodern version of the polymer BH is filled with closed strings [58, and so any excitation "sees" a $(1+1)$ dimensional geometry.
} 


\section{The postmodern perspective}

Just like a skeptic, a postmodernist is determined to eliminate the singularity from her portrait of the $\mathrm{BH}$ interior. The difference here is that the postmodernist is willing to extend the bounds of conventional physics; for instance, quantum physics extending gravity beyond classical GR, exotic matter extending field theory beyond the SM or likely both. The prototypical example of the postmodern perspective is the string-theory inspired fuzzball model [26]. Other prominent examples are 't Hooft's BHs without interiors [28, 29], the graviton-condensate model of Dvali and Gomez [30] and, depending on its classification, Maldacena and Susskind's ER=EPR proposal. Except for a brief discussion at the end of the section, much of our focus will again be on the polymer model of the BH, although now from the postmodernist's perspective. The polymer model, just like the others, relies on properties of of string theory and describes deviations over horizon-sized scales, but it also provides a direct link to the discussion in the previous section. Another example of the link between a skeptic and a postmodernist perspective is described in [66]. There, the graviton condensate model is mapped on the gravastar model.

The polymer model grew out of the notion that the BH interior must be in a nonclassical state, even at times before the Page time. This claim has been a common theme in some of our recent work, beginning with [67], but the most explicit argument is presented in [68]. There, it is shown that the $\mathrm{BH}$ radiation is in a highly quantum state when expressed in terms of the Fock (or occupation number) basis of asymptotic quantum fields. It follows that the purifier of the radiation - the $\mathrm{BH}$ interior is of a similarly quantum nature when expressed in the corresponding basis (this is also shown explicitly in [68]). Our conclusion is that a geometrical description of the interior in terms of a semiclassical metric is not feasible. Simply put, the quantum 
fluctuations in the metric would be as least as large as the corresponding expectation values.

The premise of the polymer model is that the $\mathrm{BH}$ interior consists of highly excited, long, closed, interacting strings [69, 58]. The primary motivation is that the natural distribution of matter for a highly quantum interior happens to have the same equation of state as a collection of long, closed strings when heated to just above the Hagedorn temperature, as included below for completeness. What closes this circle of logic is that such strings have a high density of states and, as such, are subject to exceptionally large quantum fluctuations [70].

The equation of state for this high-temperature string phase is famously $p=\rho$ [71. In such a phase, the entropy density $s$ and temperature $T$ are related to the energy density, in string units, as $s=\sqrt{\rho}$ and $1 / T=d s / d \rho=s / 2 \rho$. Then $p=-\rho+s T=+\rho$ which is, of course, consistent with the thermodynamic relation $s T=p+\rho$. Therefore, $s$ is as large as it could be in comparison to $\rho$, implying entropic dominance [72] and correspondingly large quantum fluctuations.

How does this all connect to the skeptic's description of the polymer model? Recalling Eq. (4) but now with positive pressure $\left(l_{P}=\sqrt{G}\right.$ is the Planck length),

$$
p_{r}=+\rho=\frac{1}{8 \pi l_{P}^{2} r^{2}}
$$

and using the above reasoning to obtain the entropy density,

$$
s=\sqrt{\frac{2 \pi \rho}{l_{P}^{2}}}=\frac{1}{2 r l_{P}^{2}},
$$

we find that the mass and entropy inside a sphere of radius $R<R_{S}$ are respectively

$$
m(r<R)=\frac{1}{2} \frac{R}{l_{P}^{2}}
$$


and

$$
S(r<R)=\pi \frac{R^{2}}{l_{P}^{2}} .
$$

These are indeed the expected scaling relations given that each spherical slice is supposed to act like a BH horizon (just like for the skeptic). However, because these configurations lack a reliable description in terms of a (semi)classical metric, the coordinate $r$ should be thought of as a fiducial coordinate rather than the radial coordinate of a spherically symmetric, classical geometry.

It is worth noting some prominent features of the polymer model that are evident when the pressure is regarded as positive [58, 73]: The outer boundary behaves classically like a horizon simply because the interaction strength of the strings scales with $\hbar$. However, when Plank's constant is turned on, then bits of string will leak out with an energy and at a rate that matches those of Hawking particles. 5 In addition, this model has been shown to saturate both the maximally allowed value of the Lyapunov exponent and the minimally allowed ratio of shear viscosity to entropy density, as first conjectured in [74] and [75] respectively. Most of this analysis would be of little interest to a skeptic or a modernist; both of whom would insist on a well-defined metric for the interior.

The radial pressure is, of course, a significant difference between the skeptic and postmodernist version of the polymer model: It has gone from maximally negative $p_{r}=-\rho$ in the former case to maximally positive $p_{r}=+\rho$ in the latter. Either way, the transverse pressure $p_{\perp}$ is vanishing on average. Our viewpoint is that the latter version provides the more fundamental description; however, the geometry can not be sourced by an energy-momentum tensor with $p_{r}=+\rho$ because there is formally no room for entropy in the Einstein equations. This is yet another manifestation of

\footnotetext{
${ }^{5}$ The string bits are subject to Hagedorn-scale excitations inside. However, the average of the net bound energy per bit is that of a typical Hawking mode.
} 
the inadequacy of classical geometry (or even semiclassical geometry for that matter) when it is used to describe a maximally entropic configuration.

The deviation between the two pressures, even if maximally large, is somewhat superficial; the real difference is one of perspectives. This point has already been made in [45], but we are now well positioned to sharpen this distinction: The skeptic accepts that the interior could contain matter but is otherwise agnostic about its nature. He is simply describing the interior in terms of pressure $p$ and energy density $\rho$ and insists that these be chosen in a way that evades the Buchdahl bound. He soon finds out that the only viable loophole is a maximally negative pressure. And, although this implies that the entropy is vanishing, he neglects this "minor detail" as his interest is classical gravity and not quantum thermodynamics. Moreover, given that there is no spontaneous emission of radiation in the skeptic's version, a vanishing entropy seems reasonable. In contrast, the postmodernist quite willingly accepts the exotic stringy description of the interior and could use it to reproduce the above analysis, including the positive pressure. The sign of the pressure, however, is not a problem because for this observer, lacking an adequate geometry, the Buchdahl bound is not relevant. This divergence in perspectives would be similar to a scenario in which the accelerated expansion of the Universe is really due to undetectable entropic matter and not a cosmological constant [76].

Although the two versions of the polymer model are different in some ways, they are quite similar with regard to the propagation of signals in the interior. In both cases, all waves propagate at the speed of light because the magnitude of the pressure is maximal, $|p / \rho|=v^{2} / c^{2}$. One can also understand this from physically motivated perspectives. In the skeptic's picture of the polymer model, the entire interior has to be null and, therefore, so too must every geodesic. Meanwhile, the postmodernist views the interior as being filled with long, closed strings or, to some reasonable 


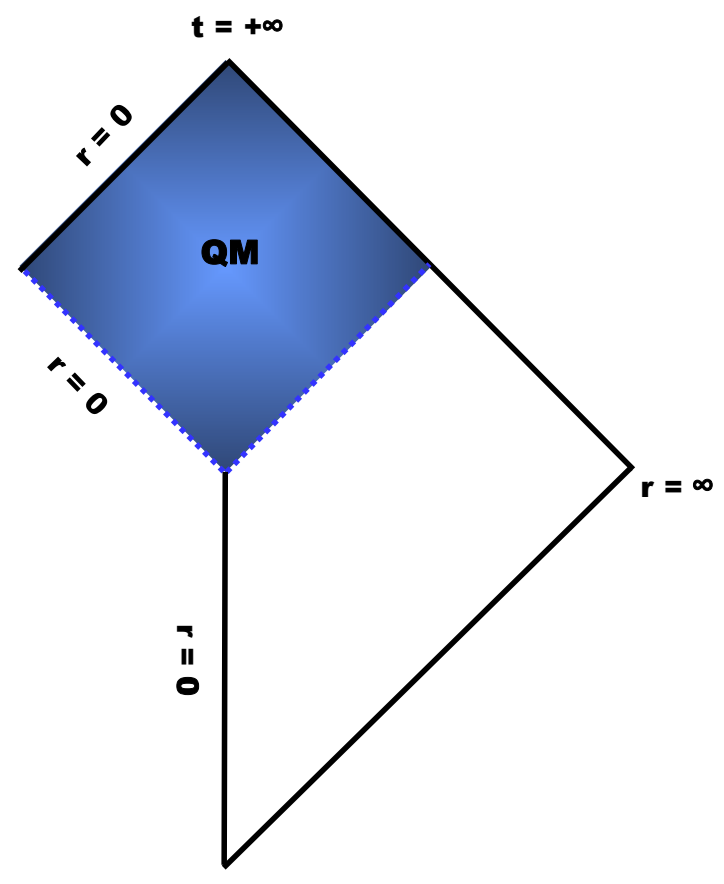

Figure 6: Penrose diagram of the polymer model of a regular BH from a postmodern perspective. See Fig. 5 for the diagram representing the skeptic's perspective of the same model. The above diagram should also be viewed as part of an extended diagram.

approximation, a single long loop of string. As this is effectively a $1+1$-dimensional entity, it follows that all geodesics are,locally, maximally focused and any propagation must then follow the same path as a null ray.

The postmodernists still need to explain why the polymer does not collapse, as the negative-pressure argument no longer applies. These observers rather put an emphasis on the exotic nature of the interior matter and discover that some simple calculations reveal a classically stable, Schwarzschild-sized state [58]. They attribute 
this stability to the fact that the entropically favorable state for the strings is one of a few long loops occupying a region of space which is the same size as their random walks. To see this, consider that a long string of length $L$ has an entropy of $S_{\text {long }}=L / l_{s}$ (and a random-walk size of $\sqrt{S_{\text {long }}}$ ), whereas $N$ shorter strings each of length $L / N$ have a total entropy of $S_{\text {short }}=\frac{\ln N}{N} S_{\text {long }}$, which is much smaller than $L / l_{s}$ even when $N$ is somewhat larger than one. For example, consider the case that the total entropy is that of a solar-mass $\mathrm{BH}, S \sim 10^{76}$. Then the difference between the entropy of a single long string and that of two half-length strings is $\frac{\ln 2}{2} S$, which is of order of $S$ itself. Moreover, this maximally large entropy explains why an arbitrary state of matter would transition into a hot stringy soup in the first place.

How the polymer evades the causality paradox - from the perspective of an external observer - proved to be the toughest nut to crack. Of course, an observer who accepts the complete picture, including the interaction rate of the strings, might not be so concerned. But can this issue be be reconciled for the less informed as well? Fortunately, this matter was recently resolved in [77] as will now be explained.

We will start with BHs out of equilibrium, paralleling part of the discussion about the skeptic's perspective from the previous section. It will be assumed that the $\mathrm{BH}$ has been excited by some external perturbation with a monopole component that is either negligibly small or negative; both cases are physically acceptable as observed in [77]. Such disturbances will depress some part of the horizon via tidal effects [78, 79, 63]; in which case, an interior mode could very well be exposed to the outside and thus have the opportunity to escape. From an external perspective, a mode of Hawking radiation could just as well have been "born" in the exterior spacetime where causality is certainly not an issue.

But what about a $\mathrm{BH}$ in equilibrium which is not exposed to any deforming sources; should it not be permitted to radiate? Recall that, from the skeptic's per- 
spective, such a $\mathrm{BH}$ does not radiate. But the postmodernists want to include Hawking radiation in the picture, which means explaining how a $\mathrm{BH}$ in equilibrium can overcome the causality paradox. For this, they can rely on quantum fluctuations in the horizon position, as the outer boundary of an ultracompact object would normally experience Planckian-sized fluctuations at the very least [73]. Probing a sub-Planckian length scale would turn the probing apparatus into a $\mathrm{BH}$, so that one could never know any position more precisely than $l_{P}$. And, as explained in [77], a Planckian-sized fluctuation is exactly what is needed. This is because a mode of wavelength $l_{P}$ will be redshifted by a factor of $R_{S} / l_{P}$ by the time it reaches an asymptotic observer.

The previous argument would seem to apply to just about any model of the interior. But, in actuality, one needs a fluid-filled object to account for the perpetual supply of near-horizon modes or, failing that, at least a limited concentration of matter in the center. This argument is then negated as far as a modernist is concerned. Meanwhile, it is the lack of quantum fluctuations in the interior that makes it problematic for a skeptic to use. This is certainly the case for the negative-pressure version of the polymer (which is completely resistant to such fluctuations), but also in general insofar as regularized BHs are rendered inconsistent by quantum effects.

\section{The minimalist perspective}

A subgroup of the postmodernists are those who would do away with the interior altogether, a school of thought which is akin to minimalism. This would include Mathur's fuzzball model, according to his interpretation of the physical picture in [80] and [81]. More emphatically, 't Hooft argues away the interior by asserting that

incoming particles can be exchanged for outgoing ones before the energies of the 
former are red-shifted to Planckian scales; a process which he refers to as "firewall transformations" [28, 29]. 't Hooft also argues on behalf of an antipodal identification between the two exterior regions in the extended Penrose diagram. In this way, an incoming particle is transmuted at the bifurcation surface and emerges in the opposite region without traversing the interior. The two interior regions are thus rendered irrelevant and can be discarded. The reason that this model is classified under postmodernism is because both critical features are motivated by the requirement of preserving quantum-mechanical principles. It will be argued elsewhere that this description of the $\mathrm{BH}$ and that of the polymer model share much in common [61, which can be viewed as Hawking's principle of ignorance at work.

\section{Conclusion}

Given Hawking's principle of ignorance and the opaque nature of a BH horizon, different observers do not necessarily have to agree on what lies within. With this idea in mind, we have considered the perspectives of three different external observers: the modernist, who expects something akin to the BHs of GR, the skeptic, who would prefer to eliminate gravitational collapse but without giving up on GR and the postmodernist, who is willing to rely on exotic physics to achieve a self-consistent quantum-mechanical description which is devoid of collapse. The preferences of each of the three observers are compared in Table 1.

But, out of the three, only the postmodern perspective can consistently explain what is known (so far) about BH physics. And, although the discussion has been emphasizing the issues of gravitational collapse, reproducing $\mathrm{BH}$ thermodynamics and the causality paradox, any quantum-based, non-collapsing model of the interior is guaranteed to satisfy the quantum laws of unitary evolution and the strong sub- 


\begin{tabular}{|l|c|c|c|c|c|}
\hline Observer & Regular & TD & QM & GR & SM \\
\hline Modernist & No & Yes & No & Yes & Yes \\
Skeptic & Yes & No & No & Yes & No \\
Postmodernist & Yes & Yes & Yes & No & No \\
\hline
\end{tabular}

Table 1: Comparison between the three observers. The new abbreviations TD and QM stand for thermodynamics and quantum mechanics, respectively.

additivity of entropy. Meaning that the notorious firewall problem is similarly of no concern to a postmodernist. The singularity is resolved by extending the quantumgravitational scale all the way up to the horizon.

Taking the principle of ignorance and the one-way nature of the horizon seriously, one might wonder if anything could be said definitively about the BH interior. Fortunately, the ever-growing body of gravitational-wave data casts a whole new light on this prospect. After all, Hawking's principle stipulates that one's explanation must be consistent with what is already known, and so each new data point further constrains the collection of permissible stories. The reason that gravitational waves are particularly valuable in this way is because they represent the product of out-ofequilibrium physics - for instance, the violent merger of the two BHs in a binary system - whereas the more traditional aspects of BH physics (such as Hawking radiation and the area-entropy law) assumes systems at or near equilibrium. It is our expectation that any new physics should indeed leave just such a signature in the gravitational-wave data [82]. Also see [83, 84] for a more general discussion.

One of the initial motivations for this paper was to better understand how the inside of a polymer BH can have two different equations of state [45], even though 
any relevant observer would have to be located in the exterior. But, as we now understand, the BH entropy may or may not be hidden depending on how the observer explains the stability of the polymer against gravitational collapse. It is our expectation that a similar dichotomy applies to the accelerated expansion of the Universe [76].

Although a main part of our focus was on the polymer BH, it should be noted that models of BH-like objects are rampant in the literature (see [83] for a thorough yet incomplete catalog). But the takeaway point should be that exotic physics, by which we mean beyond the SM and GR, is necessary for a consistent description of evaporating BHs. Either way, the polymer BH would appear to be a viable model. But there will come a time when such speculations no longer matter, as it does seem inevitable that any given proposal will be seriously tested by data form future gravitational-wave experiments. It is then just as inevitable that, for the vast majority of $\mathrm{BH}$ models, the current state of ignorance will indeed be bliss.

\section{Acknowledgments}

The research of RB was supported by the Israel Science Foundation grant no. 1294/16. The research of AJMM received support from an NRF Incentive Funding Grant 85353

and a Rhodes University Discretionary Grant RD51/2018. AJMM thanks Ben Gurion University for their hospitality during his visit.

\section{References}

[1] D. Finkelstein, "Past-Future Asymmetry of the Gravitational Field of a Point Particle," Phys. Rev. 110, 965 (1958). 
[2] J. A. Wheeler, "Geons," Phys. Rev. 97, 511 (1955).

[3] H. Buchdahl, "General Relativistic Fluid Spheres," Phys. Rev. 116, 1027 (1959).

[4] S. Chandrasekhar "Dynamical Instability of Gaseous Masses Approaching the Schwarzschild Limit in General Relativity," Phys. Rev. Lett. 12, 114 (1964).

[5] S. Chandrasekhar, "The Dynamical Instability of Gaseous Masses Approaching the Schwarzschild Limit in General Relativity," Astrophys. J. 140, 417 (1964).

[6] H. Bondi, "Massive spheres in general relativity," Proc. Roy. Soc. Lond. A 282, $303(1964)$.

[7] A. K. Raychaudhuri, "Relativistic cosmology I," Phys. Rev. 98, 1123 (1955).

[8] A. B. Komar, "Necessity of Singularities in the Solution of the Field Equations of General Relativity," Phys. Rev. 104, 544 (1956).

[9] R. Penrose, "Gravitational Collapse and Space-Time Singularities," Phys. Rev. Lett. 14, 57 (1965).

[10] S. W. Hawking and R. Penrose, "The singularities of gravitational collapse and cosmology," Proc. R. Soc. Lond. A 314, 529 (1970).

[11] J. D. Bekenstein, "Black holes and entropy," Phys. Rev. D 7, 2333 (1973).

[12] S. W. Hawking, "Black hole explosions," Nature 248, 30 (1974); "Particle creation by black holes," Comm. Math. Phys. 43, 199 (1975).

[13] S. W. Hawking, "Breakdown of predictability in gravitational collapse," Phys. Rev. D 14, 2460 (1976). 
[14] L. Susskind, L. Thorlacius and J. Uglum, "The Stretched horizon and black hole complementarity," Phys. Rev. D 48, 3743 (1993) [hep-th/9306069].

[15] C. R. Stephens, G. 't Hooft and B. F. Whiting, "Black hole evaporation without information loss," Class. Quant. Grav. 11, 621 (1994) [gr-qc/9310006].

[16] L. Susskind and L. Thorlacius, "Gedanken experiments involving black holes," Phys. Rev. D 49, 966 (1994) [hep-th/9308100].

[17] N. Itzhaki, "Is the black hole complementarity principle really necessary?" arXiv:hep-th/9607028.

[18] S. D. Mathur, "What Exactly is the Information Paradox?," Lect. Notes Phys. 769, 3 (2009) [arXiv:0803.2030 [hep-th]].

[19] S. L. Braunstein, S. Pirandola and K. Zyczkowski, "Entangled black holes as ciphers of hidden information," Physical Review Letters 110, 101301 (2013) [arXiv:0907.1190 [quant-ph]].

[20] D. Marolf and J. Polchinski, "Gauge/Gravity Duality and the Black Hole Interior," Phys. Rev. Lett. 111, 171301 (2013) [arXiv:1307.4706 [hep-th]].

[21] S. D. Mathur, "What does strong subadditivity tell us about black holes?," Nucl. Phys. Proc. Suppl. 251-252, 16 (2014) [arXiv:1309.6583 [hep-th]].

[22] A. Almheiri, D. Marolf, J. Polchinski and J. Sully, "Black Holes: Complementarity or Firewalls?," JHEP 1302, 062 (2013) [arXiv:1207.3123 [hep-th]].

[23] A. Almheiri, D. Marolf, J. Polchinski, D. Stanford and J. Sully, "An Apologia for Firewalls," JHEP 1309, 018 (2013) [arXiv:1304.6483 [hep-th]]. 
[24] L. Susskind, "Trouble for remnants," hep-th/9501106.

[25] P. Chen, Y. C. Ong and D. h. Yeom, "Black Hole Remnants and the Information Loss Paradox," Phys. Rept. 603, 1 (2015) [arXiv:1412.8366 [gr-qc]].

[26] S. D. Mathur, "The Quantum structure of black holes," Class. Quant. Grav. 23, R115 (2006) [hep-th/0510180].

[27] K. Skenderis and M. Taylor, "The fuzzball proposal for black holes," Phys. Rept. 467, 117 (2008) [arXiv:0804.0552 [hep-th]].

[28] G. 't Hooft, "Black hole unitarity and antipodal entanglement," Found. Phys. 46, no. 9, 1185 (2016) [arXiv:1601.03447 [gr-qc]].

[29] G. 't Hooft, "The Firewall Transformation for Black Holes and Some of Its Implications," Found. Phys. 47, no. 12, 1503 (2017) [arXiv:1612.08640 [gr-qc]].

[30] G. Dvali and C. Gomez, "Black Hole's Quantum N-Portrait," Fortsch. Phys. 61, 742 (2013) [arXiv:1112.3359 [hep-th]].

[31] S. D. Mathur, "Resolving the black hole causality paradox," arXiv:1703.03042 [hep-th].

[32] J. A. Wheeler, "Geometrodynamics and the Issue of the Final State" in Relativity, Groups, and Topology, Lectures Delivered at Les Houches During the 1963 Session of the Summer School of Theoretical Physics, University of Grenoble, C. DeWitt and B. DeWitt, (Eds.), pp. 317-501, Gordon and Breach, New York, 1964.

[33] P. C. W. Davies, "Thermodynamics of black holes," Rept. Prog. Phys. 41, 1313 (1978). 
[34] D. N. Page, "Average entropy of a subsystem," Phys. Rev. Lett. 71, 1291 (1993) [gr-qc/9305007]; "Information in black hole radiation," Phys. Rev. Lett. 71, 3743 (1993) [hep-th/9306083].

[35] A. Akil, O. Dahlsten and L. Modesto, "Entanglement swapping in black holes: restoring predictability," arXiv:1805.09573 [hep-th].

[36] G. T. Horowitz and J. M. Maldacena, "The Black hole final state," JHEP 0402, 008 (2004) [hep-th/0310281].

[37] D. Gottesman and J. Preskill, "Comment on 'The Black hole final state'," JHEP 0403, 026 (2004) [hep-th/0311269].

[38] J. Maldacena and L. Susskind, "Cool horizons for entangled black holes," Fortsch. Phys. 61, 781 (2013) [arXiv:1306.0533 [hep-th]].

[39] G. 't Hooft, "On the quantum structure of a black hole," Nucl. Phys. B 256, 727 (1985).

[40] S. A. Hayward, "Formation and evaporation of regular black holes," Phys. Rev. Lett. 96, 031103 (2006) [gr-qc/0506126].

[41] V. P. Frolov, M. A. Markov and V. F. Mukhanov, "Black Holes as Possible Sources of Closed and Semiclosed Worlds," Phys. Rev. D 41, 383 (1990).

[42] P. O. Mazur and E. Mottola, "Gravitational condensate stars: An alternative to black holes," arXiv:gr-qc/0109035.

[43] C. Barcelo, S. Liberati, S. Sonego and M. Visser, "Fate of gravitational collapse in semiclassical gravity," Phys. Rev. D 77, 044032 (2008) [arXiv:0712.1130 [gr$q \mathrm{qc}]$. 
[44] R. Carballo-Rubio, "Stellar equilibrium in semiclassical gravity," Phys. Rev. Lett. 120, no. 6, 061102 (2018) [arXiv:1706.05379 [gr-qc]].

[45] R. Brustein and A. J. M. Medved, "Resisting collapse: How matter inside a black hole can withstand gravity," arXiv:1805.11667 [hep-th].

[46] F. P. Gonzalez-Diaz, "The space-time metric inside a black hole," Lett. Nuovo Cimento 32, 161 (1981).

[47] M. R. Mbonye and D. Kazanas, "Nonsingular black hole model as a possible end product of gravitational collapse," Phys. Rev. D 72, 024016 (2005); "Can Gravitational Collapse Sustain Singularity-Free Trapped Surfaces?," Int. J. Mod. Phys. D 17, 165 (2008).

[48] E. Guendelman, A. Kaganovich, E. Nissimov and S. Pacheva, "Non-singular black holes from gravity-matter-brane lagrangians," Int. J. Mod. Phys. A 25, 1571 (2010) [arXiv:0908.4195 [hep-th]].

[49] V. P. Frolov, "Notes on nonsingular models of black holes," Phys. Rev. D 94, no. 10, 104056 (2016) [arXiv:1609.01758 [gr-qc]].

[50] E. Spallucci and A. Smailagic, "Regular black holes from semi-classical down to Planckian size," Int. J. Mod. Phys. D 26, no. 07, 1730013 (2017) [arXiv:1701.04592 [hep-th]].

[51] D. Malafarina, "Classical collapse to black holes and quantum bounces: A review," Universe 3, no. 2, 48 (2017) [arXiv:1703.04138 [gr-qc]].

[52] J .M. Bardeen, "Non-singular general-relativistic gravitational collapse," in Proceedings of International Conference GR5, p. 174 (Tbilisi, USSR,1968). 
[53] E. Ayon-Beato and A. Garcia, "Regular black hole in general relativity coupled to nonlinear electrodynamics," Phys. Rev. Lett. 80, 5056 (1998) [gr-qc/9911046].

[54] V. P. Frolov and G. A. Vilkovisky, "Spherically Symmetric Collapse in Quantum Gravity," Phys. Lett. 106B, 307 (1981); "Quantum gravity removes classical singularities and shortens the life of black holes," in The Second Marcel Grossmann Meeting on the Recent Developments of General Relativity, p. 455 (Trieste, Italy, 1979).

[55] V. P. Frolov and A. Zelnikov, "Quantum radiation from an evaporating nonsingular black hole," Phys. Rev. D 95, no. 12, 124028 (2017) [arXiv:1704.03043 [hep-th]].

[56] R. Carballo-Rubio, F. Di Filippo, S. Liberati, C. Pacilio and M. Visser, "On the viability of regular black holes," JHEP 1807, 023 (2018) [arXiv:1805.02675 [gr-qc]].

[57] P. O. Mazur and E. Mottola, "Surface tension and negative pressure interior of a non-singular black hole," Class. Quant. Grav. 32, no. 21, 215024 (2015) [arXiv:1501.03806 [gr-qc]].

[58] R. Brustein and A. J. M. Medved, "Black holes as collapsed polymers," Fortsch. Phys. 65, 0114 (2017) [arXiv:1602.07706 [hep-th]].

[59] E. I. Guendelman and A. I. Rabinowitz, "Hedgehog compactification," Phys. Rev. D 47, 3474 (1993) Erratum: [Phys. Rev. D 48, 2961 (1993)].

[60] E. I. Guendelman and A. Rabinowitz, "The Gravitational field of a hedgehog and the evolution of vacuum bubbles," Phys. Rev. D 44, 3152 (1991). 
[61] R. Brustein and A. J. M. Medved, work in progress.

[62] R. Brustein, A. J. M. Medved and Y. Zigdon, work in progress.

[63] R. Brustein and Yotam Sherf, work in progress.

[64] N. Itzhaki, "Stringy instability inside the black hole," JHEP 1810, 145 (2018) [arXiv:1808.02259 [hep-th]].

[65] R. M. Wald, "Black hole entropy is the Noether charge," Phys. Rev. D 48, no. 8, R3427 (1993) [gr-qc/9307038].

[66] F. Cunillera and C. Germani, "The GrossPitaevskii equations of a static and spherically symmetric condensate of gravitons," Class. Quant. Grav. 35, no. 10, 105006 (2018) [arXiv:1711.01282 [gr-qc]].

[67] L. Alberte, R. Brustein, A. Khmelnitsky and A. J. M. Medved, "Density matrix of black hole radiation," JHEP 1508, 015 (2015) [arXiv:1502.02687 [hep-th]].

[68] R. Brustein, A. J. M. Medved and Y. Zigdon, "The state of Hawking radiation is non-classical," JHEP 1801, 136 (2018) [arXiv:1707.08427 [hep-th]].

[69] R. Brustein and A. J. M. Medved, "Quantum state of the black hole interior," JHEP 1508, 082 (2015) [arXiv:1505.07131 [hep-th]].

[70] N. Deo, S. Jain and C.-I. Tan, "String distributions above Hagedorn energy density," Phys. Rev. D 40, 2626 (1989).

[71] J. J. Atick and E. Witten, "The Hagedorn transition and the number of degrees of freedom in string theory," Nucl. Phys. B 310, 291 (1988). 
[72] R. Brustein and G. Veneziano, "A Causal entropy bound," Phys. Rev. Lett. 84, 5695 (2000) [hep-th/9912055].

[73] R. Brustein and A. J. M. Medved, "Emergent horizon, Hawking radiation and chaos in the collapsed polymer model of a black hole," Fortsch. Phys. 65, no. 2, n/a, 1600116 (2017) [arXiv:1607.03721 [hep-th]].

[74] J. Maldacena, S. H. Shenker and D. Stanford, "A bound on chaos," JHEP 1608, 106 (2016) [arXiv:1503.01409 [hep-th]].

[75] P. Kovtun, D. T. Son and A. O. Starinets, "Viscosity in strongly interacting quantum field theories from black hole physics," Phys. Rev. Lett. 94, 111601 (2005) [hep-th/0405231].

[76] R. Brustein and A. J. M. Medved, work in progress.

[77] R. Brustein and A. J. M. Medved, "Quantum hair of black holes out of equilibrium," Phys. Rev. D 97, no. 4, 044035 (2018) [arXiv:1709.03566 [hep-th]].

[78] J. B. Hartle, "Tidal Friction in Slowly Rotating Black Holes," Phys. Rev. D 8, 1010 (1973).

[79] S. O'Sullivan and S. A. Hughes, "Strong-field tidal distortions of rotating black holes: Formalism and results for circular, equatorial orbits," Phys. Rev. D 90, no. 12, 124039 (2014) Erratum: [Phys. Rev. D 91, no. 10, 109901 (2015)] [arXiv:1407.6983 [gr-qc]].

[80] S. D. Mathur, "A model with no firewall," arXiv:1506.04342 [hep-th].

[81] S. D. Mathur, "What does the information paradox say about the universe?," arXiv:1812.11641 [hep-th]. 
[82] R. Brustein, A. J. M. Medved and K. Yagi, "When black holes collide: Probing the interior composition by the spectrum of ringdown modes and emitted gravitational waves," Phys. Rev. D 96, no. 6, 064033 (2017) [arXiv:1704.05789 [gr-qc]].

[83] V. Cardoso and P. Pani, "Tests for the existence of horizons through gravitational wave echoes," Nat. Astron. 1, 586 (2017) [arXiv:1709.01525 [gr-qc]]; "The observational evidence for horizons: from echoes to precision gravitational-wave physics," arXiv:1707.03021 [gr-qc].

[84] R. Carballo-Rubio, F. Di Filippo, S. Liberati and M. Visser, "Phenomenological aspects of black holes beyond general relativity," Phys. Rev. D 98, no. 12, 124009 (2018) doi:10.1103/PhysRevD.98.124009 [arXiv:1809.08238 [gr-qc]]. 